\title{
Combining biologic and phototherapy treatments for psoriasis: safety, efficacy, and patient acceptability
}

\author{
Benjamin Farahnik' \\ Viraat Patel $^{2}$ \\ Kourosh Beroukhim ${ }^{3}$ \\ Tian Hao Zhu ${ }^{4}$ \\ Michael Abrouk ${ }^{2}$ \\ Mio Nakamura ${ }^{5}$ \\ Rasnik Singh ${ }^{3}$ \\ Kristina Lee ${ }^{5}$ \\ Tina Bhutani ${ }^{5}$ \\ John $\mathrm{Koo}^{5}$
}

'University of Vermont College of Medicine, Burlington, VT; ${ }^{2} \mathrm{School}$ of Medicine, University of California, Irvine, ${ }^{3}$ David Geffen School of Medicine, University of California, Los Angeles, ${ }^{4}$ University of Southern California Keck School of Medicine, Los Angeles, ${ }^{5}$ Department of Dermatology, Psoriasis and Skin Treatment Center, University of California, San Francisco, CA, USA

Correspondence: Benjamin Farahnik Department of Dermatology, University of California, San Francisco 515 Spruce St, San Francisco, CA 94II8, USA

Tel +I 3109233278

Fax + I 4I5 5024126

Email Benjamin.farahnik@med.uvm.edu
This article was published in the following Dove Press journal:

Psoriasis: Targets and Therapy

28 July 2016

Number of times this article has been viewed

Background: The efficacy and safety of biologic and phototherapy in treating moderate-tosevere psoriasis is well known. However, some patients may not respond well to biologic agents or phototherapy on their own and may require combination therapy. Skillfully combining a biologic agent and phototherapy may provide an additive improvement without much increase in risks.

Objective: To summarize the current state of evidence for the efficacy and safety of combining biologics with phototherapy in the treatment of moderate-to-severe plaque psoriasis.

Methods: We conducted an extensive search on Pubmed database for English language literature that evaluated the use of a combination of biologic and phototherapy for the treatment of moderate-to-severe psoriasis through January 2016. The search included the following keywords: psoriasis, etanercept, adalimumab, infliximab, ustekinumab, biologics, phototherapy, and combination therapy.

Results: The primary literature included randomized controlled trials, a head-to-head study, open-label controlled and uncontrolled trials, case series, and case reports. Etanercept was used in over half of the reported cases, but other biologic agents used included ustekinumab, adalimumab, and infliximab. The vast majority of phototherapy was narrowband ultraviolet B (NBUVB) radiation. Most cases reported enhanced improvement with combination therapy. Serious adverse events throughout the study duration were reported in $<3 \%$ of the patients. Long-term adverse events cannot be excluded.

Conclusion: Combination of biologic and phototherapy appears to be a viable clinical strategy in the treatment of moderate-to-severe psoriasis not responsive to monotherapy, despite limitations in the data available. NBUVB in combination with biologics appears to be especially effective. However, the long-term impact of these combinations is yet to be determined.

Keywords: psoriasis, biologics, phototherapy, UVB, UVA, combination therapy

\section{Introduction}

Psoriasis is a common chronic inflammatory skin condition with a worldwide prevalence of $0.5 \%$ (Asia) to $8.5 \%$ (Norway). ${ }^{1,2}$ Symptoms of psoriasis - which include redness, scaling, flaking, pruritus, skin tightness, pain, and bleeding - have a significantly negative impact on patients' physical and mental functioning. ${ }^{3}$ Psoriasis also leads to impairment in the quality of life, psychological well-being, and work productivity. ${ }^{3,4}$ Despite the rapid development of novel treatment modalities over the past two decades, surveys conducted by the National Psoriasis Foundation reveal that a significant portion of patients with psoriasis remains undertreated relative to the severity of their disease. ${ }^{3,5}$ This is especially true for patients with moderate-to-severe plaque psoriasis, who account for $20 \%-30 \%$ of the total psoriasis population. ${ }^{2,4}$ 
US Food and Drug Administration-approved biologic agents for the treatment of psoriasis include the antitumor necrosis factor agents (etanercept, adalimumab, and infliximab), the anti-interleukin-12/23 antibody (ustekinumab), and most recently, the anti-interleukin-17 antibodies (secukinumab and ixekizumab). Biologics have significantly advanced the treatment of psoriasis, although some may experience an inadequate response ${ }^{6}$ and others may experience loss of efficacy (ie, "biologic fatigue") with long-term use. ${ }^{7}$ The combination of agents may act synergistically and is often more effective than a single agent alone. Combination therapy is a concept that uses two different agents, sometimes with reduced doses, which target specific steps in the pathogenesis of psoriasis and have distinct risk profiles. ${ }^{8}$ This may enhance efficacy and allow drug sparing, decreasing the risk of long-term cumulative toxicity from a single agent at higher doses. There is an increasing number of publications demonstrating the efficacy of combination therapy with biologic agents in moderate-to-severe psoriasis. We reviewed the safety, efficacy, and patient acceptability of combination therapy involving biologic agents and phototherapy.

\section{Methods}

We searched the PubMed database up to January 1, 2016, using the following keywords: "psoriasis" or "psoriatic arthritis" and with "biologic", "etanercept", "adalimumab", "ustekinumab", "infliximab", "combination therapy", "phototherapy", "UV phototherapy", "corticosteroids", and "topical treatment". Only English-language publications involving adult humans with moderate-to-severe psoriasis were included. Publications included were randomized controlled trials, open-label controlled and uncontrolled prospective studies, retrospective studies, case series, and case reports. The references of identified publications were also investigated for additional publications of interest. Publications on the effect on psoriatic arthritis as a primary endpoint were included if information about the effect on psoriasis were included. The authors defined combination therapy as "two therapies used concomitantly for at least 4 weeks or at least one dose of an additional systemic agent at some point during treatment." We included studies that had clinical intent to transition to another medication for safety reasons. Alefacept was not included in this review as Astellas Pharma U.S. Inc. (Northbrook, IL, USA) ceased manufacturing the drug in 2011. Efalizumab was withdrawn from the market in 2009 and thus not included. At the time of writing this manuscript, there were no combination studies involving the biologics golimumab, certolizumab, secukinumab, or ixekizumab.

\section{Results}

We found a total of ten publications assessing combination therapy involving biologics and phototherapy, with all phototherapy used being narrowband ultraviolet B (NBUVB). A total of 268 patients had been placed on combination therapy among all the trials, with an average age of 43 years (Table 1). The cohorts studied were largely similar; in general, there was evidence of benefit for the use of combination therapy in psoriasis, with eight trials (six controlled and two uncontrolled) showing enhanced clinical benefit, one controlled trial showing enhanced benefit only in those patients with high adherence to NBUVB treatment regimen, and one headto-head trial showing no benefit. Combination of NBUVB and etanercept was studied in 234 patients (Table 1). In order of frequency of study use, this was followed by adalimumab (24) and ustekinumab (ten).

Many studies demonstrated the efficacy of combination of etanercept with NBUVB with improvements in Psoriasis Area Severity Index (PASI) in previously untreated patients, ${ }^{9,10}$ and in patients who experienced an inadequate response $^{11,12}$ with etanercept alone at $50 \mathrm{mg}$ once $^{9,13}$ or twice weekly (Table 1). ${ }^{10-12,14}$ Moreover, NBUVB reduced time to clearance with etanercept $50 \mathrm{mg}$ once weekly ${ }^{9,13}$ and etanercept $50 \mathrm{mg}$ twice weekly. ${ }^{14}$ Calzavara-Pinton et al, in a randomized controlled intraindividual comparison study demonstrated that all eight patients in the study who did not achieve an adequate response with either etanercept or NBUVB monotherapy ultimately achieved PASI-75 with combination treatment (Table 1 ). ${ }^{14}$ This study validated that NBUVB or etanercept alone was not responsible for the therapeutic results.

Additionally, Lynde et al demonstrated the importance of high adherence to the NBUVB regimen for achieving significant improvement in clinical response to etanercept. ${ }^{12}$ High adherence to the NBUVB regimen was defined as missing only two or less treatments in any 4-week period. Patients missing more than two treatments in a 4-week period were considered nonadherent, and did not achieve clinically significant improvement. At 16 weeks, the proportion of patients in the high adherence group achieving PASI-90 was $42.9 \%$ for etanercept with NBUVB, compared with $3.4 \%$ for etanercept monotherapy $(P=0.018)$.

Adalimumab in combination with NBUVB therapy ${ }^{15,16}$ and ustekinumab in combination with NBUVB therapy ${ }^{17}$ were also investigated. Two studies were conducted with 


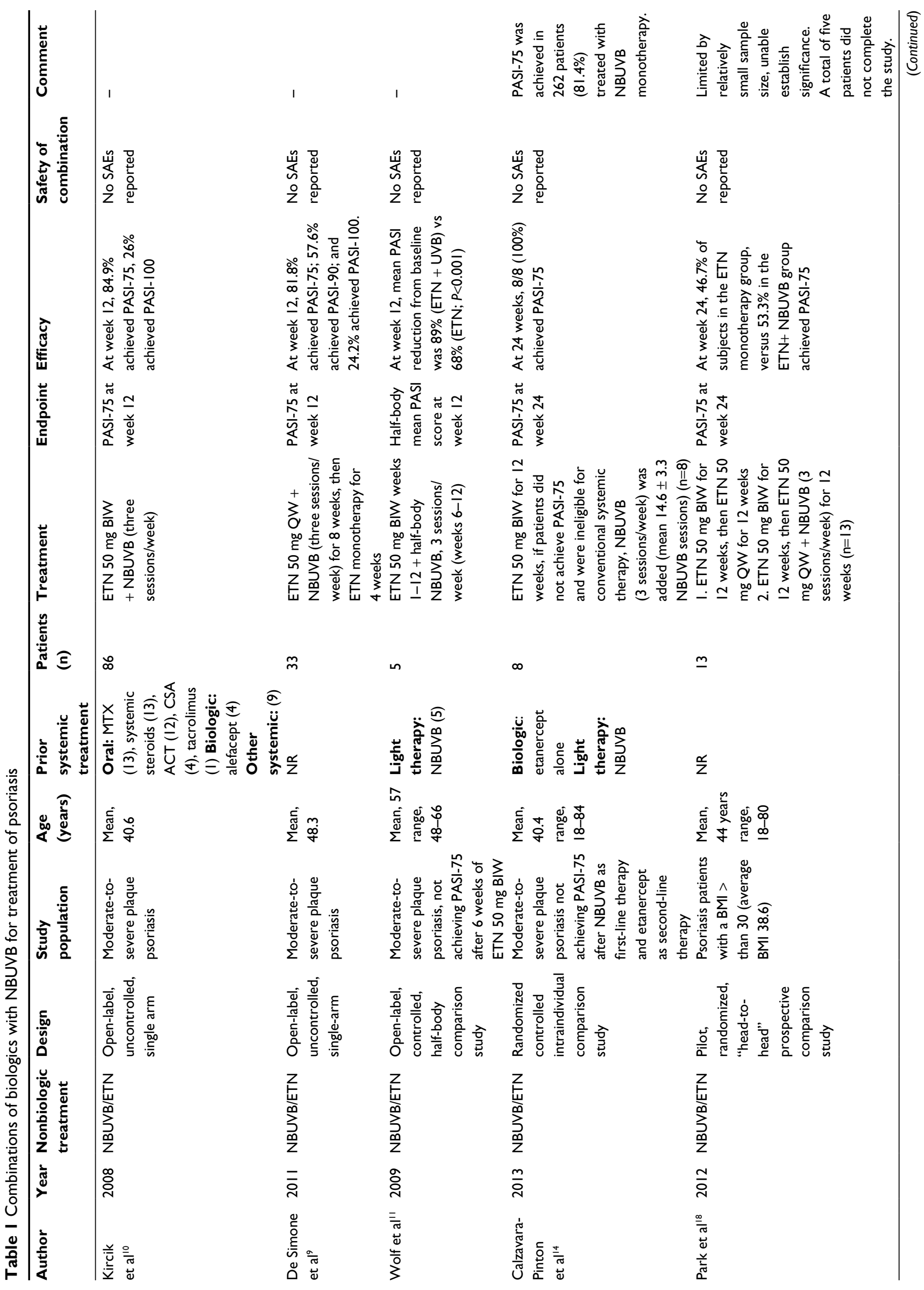




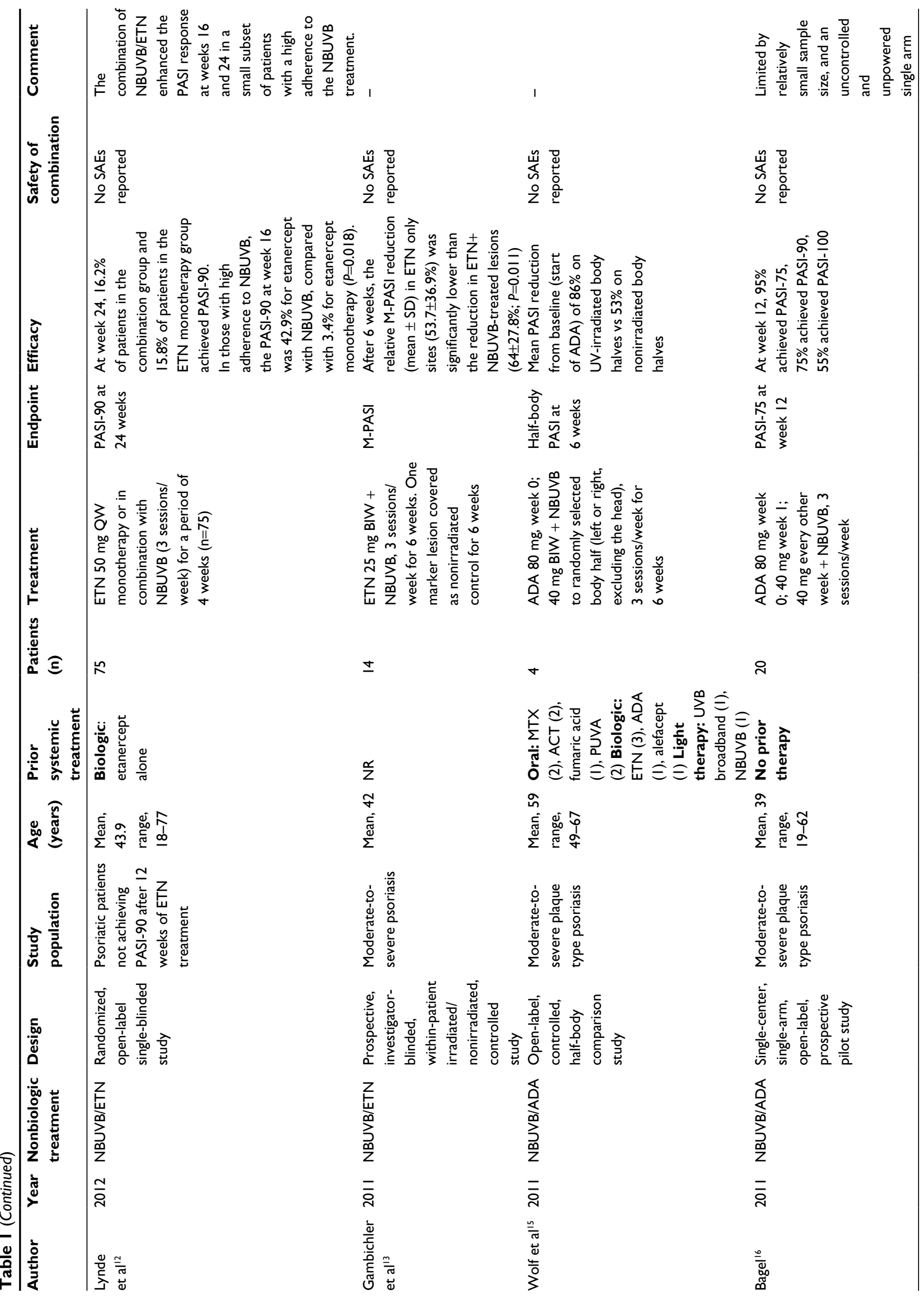




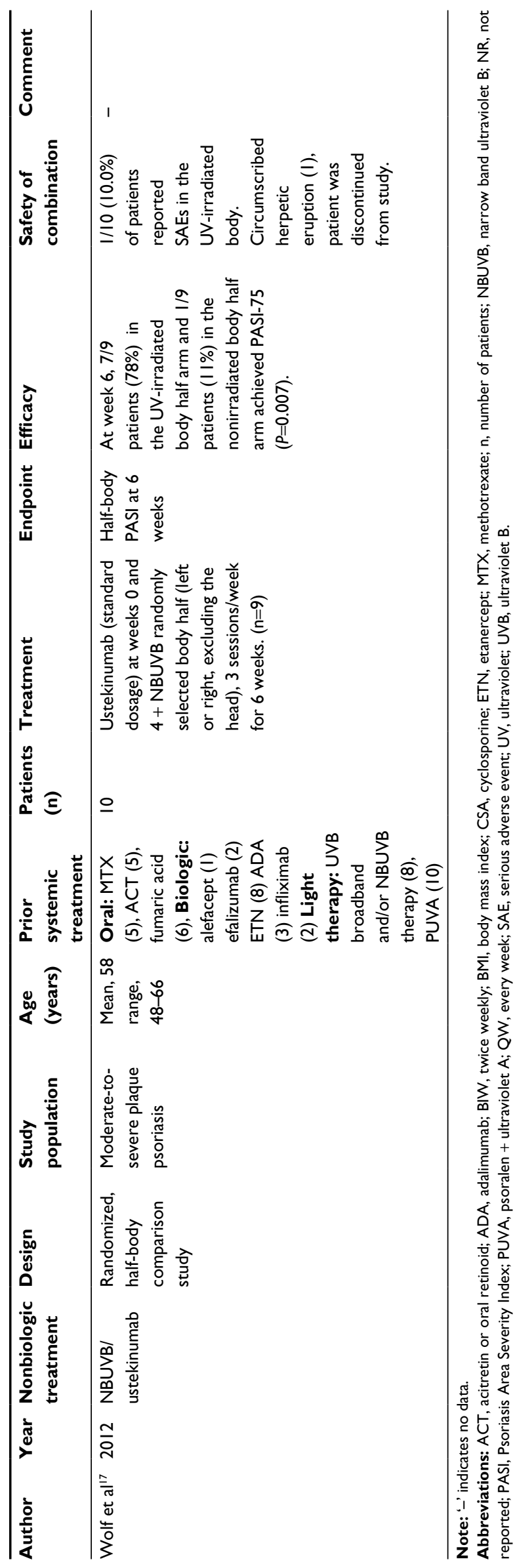

a combination of adalimumab with NBUVB. Both studies demonstrated that NBUVB significantly accelerated the response to and improved the clearance of psoriatic lesions in adalimumab-treated patients. The sole study examining ustekinumab combined with NBUVB was an intraindividual half-body comparison study conducted by Wolf et al. ${ }^{17}$ They found that PASI-75 was achieved significantly more often on the UV-irradiated body halves than the nonirradiated body halves at week 6 of the patients taking ustekinumab at a dose of 45 or $90 \mathrm{mg}$ (depending on body weight). Both combinations showed enhanced clinical improvement compared to biologic monotherapy (Table 1).

Only one study failed to establish the efficacy of combination therapy. The head-to-head pilot study by Park et al, which examined the combination treatment of NBUVB and etanercept $50 \mathrm{mg}$ twice weekly, and compared it with etanercept monotherapy, failed to demonstrate significantly enhanced improvement with combination therapy. Patients in the etanercept monotherapy, and combination of etanercept and NBUVB therapy arms had similar rates of achieving PASI-75 (46.7\% and 53.3\%, respectively); however, the small sample size limited the ability to achieve significance (Table 1). ${ }^{18}$ In addition, it is important to note that this head-to-head comparison study used psoriasis patients with a body mass index $>30$. Studies have reported a suboptimal response to etanercept in psoriasis patients with a body mass index $>30 .{ }^{19,20}$

Numerous studies have reported patient dissatisfaction as a significant barrier to optimal psoriasis treatment. ${ }^{4}$ Treatment satisfaction has been shown to predict adherence, which may affect treatment effectiveness in real-world clinical practice. ${ }^{21,22}$ Patients receiving combination treatment that was more effective in clearing psoriasis were significantly more satisfied than patients treated with monotherapy. ${ }^{21}$ In a study by Duffin et al, it was observed that patients receiving adalimumab, etanercept, ustekinumab, or NBUVB had significantly higher effectiveness scores and rates of overall satisfaction than methotrexate monotherapy or topical steroids alone. ${ }^{21}$ Interestingly enough, inconvenience has been shown to be a main factor in discontinuation of NBUVB phototherapy. ${ }^{23}$ This suggests that patient satisfaction with a treatment's effectiveness and side-effect profile may compensate for its inconvenience among patients who continue on therapy. Although no studies have directly examined patient adherence to and satisfaction of biologics in combination with phototherapy, the increased efficacy of this combination hints at the possibility of increased patient satisfaction and adherence.

In general, combination therapy involving biologics and NBUVB phototherapy was very well tolerated. The most 
common side effect noted among the studies was erythema. Nine out of the ten studies reported no serious adverse events for the combination therapy. In the study examining ustekinumab combined with NBUVB by Wolf et al, ${ }^{17}$ the serious adverse event of a herpetic eruption on the thigh, which led to treatment discontinuation, was observed. No skin cancers were reported throughout the duration of any trials. Long-term data on the development of skin cancer or other adverse events from the use of such a treatment combination has not yet been provided.

\section{Discussion}

The use of combination therapy involving a biologic agent and another form of therapy to target moderate-to-severe psoriasis is becoming more common with increased literature being released on the topic. We specifically looked at current literature that consisted of ten publications that studied combination biologic therapy with phototherapy, specifically NBUVB. The majority of publications were open-label prospective studies. In total, combination therapy was reported in 618 cases of moderate-to-severe psoriasis. The average age of these patients was 42 years, and nearly all patients had failed at least one prior systemic treatment. A serious adverse event was noted in only one patient. In general, most prospective studies used PASI-75 at week 12 or week 24 as the primary endpoint (Table 1).

The majority of available data reviewed showed that the combination of biologics with phototherapy agents had a superior efficacy compared to monotherapy in patients with moderate-to-severe psoriasis. Treatment benefit with combination therapy was demonstrated across various study designs, demonstrating that combining a biologic with phototherapy is reasonable when efficacy of monotherapy is insufficient. The largest body of evidence assessed the combination of NBUVB and etanercept. According to the nine evaluated studies, there is a reasonable evidence for the use of combination therapy with NBUVB for moderateto-severe psoriasis. Etanercept $50 \mathrm{mg}$ both once and twice weekly showed benefits, without added adverse effects.

In general, the combination of biologics with NBUVB showed good tolerability and few concerns relating to safety throughout the duration of the studies. Laboratory values and rate of adverse events for the biologics combined with phototherapy did not appear to be different from using either therapy alone. However, a potential increase in skin cancers is an important concern with the use of this combination. The immunosuppressive effect of cyclosporine, for instance, combined with phototherapy has been documented to increase the risk of skin cancer. ${ }^{24}$ Although current biologic agents are thought to be less globally immunosuppressive than cyclosporine, biologic monotherapy has been associated with a possible slightly increased risk of nonmelanoma skin cancer (NMSC) in some studies. ${ }^{25,26}$ With this in mind, the combination should be applied with caution. If possible, the combination should be limited to short durations of time for induction in difficult-to-treat cases, especially if the patient is a fair-skinned Caucasian individual. Long-term observations with NBUVB phototherapy alone have not yet demonstrated evidence of increased risk of NMSC. ${ }^{27-29}$ However, no conclusive statements can yet be made on the long-term risk of NMSC with combination treatment. Further investigation assessing long-term skin cancer risk and other adverse events in large controlled trials would be of interest.

There are some major limitations to the conclusions that can be drawn from this review. Interpreting the results is complicated by the heterogeneous study populations, small number of study subjects, and varying definitions of therapeutic success or relapse in some of the included studies investigating the efficacy and safety of combination therapy with NBUVB.

In total, 9 out of 10 studies demonstrated favorable efficacy and safety of combination therapy involving biologic and phototherapy, although the degrees of therapeutic enhancement varied. This review is significant because the subsets of patients who do not respond adequately to nonbiologic therapy are commonly encountered. Combining biologic agents with nonbiologic treatments, such as NBUVB phototherapy, broadens the armamentarium for the long-term control of moderate-to-severe psoriasis. Although no regimen involving the combination of a biologic agent and phototherapy has been approved for the management of moderate-to-severe psoriasis, the results of several relevant studies demonstrate the usefulness of such a treatment combination. Nevertheless, further studies are required to assess the long-term safety and efficacy of such combinations.

\section{Acknowledgments}

Dr John Koo is a speaker for AbbVie, Leo, and Celgene. Dr Koo conducts research for Amgen, Janssen, Novartis, Photomedex, Galderma, Pfizer, and Merck. Dr Bhutani is an advisor for Cutanea. Dr Bhutani conducts research for Abbvie, Janssen, and Merck. Drs Koo and Bhutani have no stocks, employment, or board memberships with any pharmaceutical company. The other authors (Mr Benjamin 
Farahnik, Mr Kourosh Beroukhim, Dr Mio Nakamura, Mr Michael Abrouk, Mr Henry Zhu, Ms Rasnik Singh, and Ms Kristina Lee) report no conflicts of interest.

\section{Disclosure}

John Koo is a clinical researcher for Pfizer, Amgen, Janssen and Merck, he is also a speaker for Leo Pharma, Abbvie and Celgene. Dr Bhutani conducts research for Abbvie, Janssen, and Merck. All other authors have no conflicts of interest to disclose.

\section{References}

1. Parisi R, Symmons DP, Griffiths CE, Ashcroft DM. Identification and Management of Psoriasis and Associated ComorbidiTy (IMPACT) project team. Global epidemiology of psoriasis: a systematic review of incidence and prevalence. J Invest Dermatol. 2013;133(2):377-385.

2. Rachakonda TD, Schupp CW, Armstrong AW. Psoriasis prevalence among adults in the United States. J Am Acad Dermatol. 2014;70(3):512-516.

3. Feldman SR, Malakouti M, Koo JY. Social impact of the burden of psoriasis: effects on patients and practice. Dermatol Online J. 2014;20(8).

4. Lebwohl MG, Kavanaugh A, Armstrong AW, Van Voorhees AS. US perspectives in the management of psoriasis and psoriatic arthritis: patient and physician results from the population-based Multinational Assessment of Psoriasis and Psoriatic Arthritis (MAPP) survey. Am J Clin Dermatol. 2016;17(1):87-97.

5. Krueger G, Koo J, Lebwohl M, Menter A, Stern RS, RolstadT. The impact of psoriasis on quality of life: results of a 1998 National Psoriasis Foundation patient-membership survey. Arch Dermatol. 2001;137(3):280-284.

6. Legat FJ, Hofer A, Wackernagel A, et al. Narrowband UV-B phototherapy, alefacept, and clearance of psoriasis. Arch Dermatol. 2007;143(8):1016-1022.

7. Levin EC, Gupta R, Brown G, Malakouti M, Koo J. Biologic fatigue in psoriasis. J Dermatolog Treat. 2014;25(1):78-82.

8. Lebwohl M, Menter A, Koo J, Feldman SR. Combination therapy to treat moderate to severe psoriasis. J Am Acad Dermatol. 2004;50(3):416-430.

9. De Simone C, D’Agostino M, Capizzi R, Capponi A, Venier A, Caldarola $\mathrm{G}$. Combined treatment with etanercept $50 \mathrm{mg}$ once weekly and narrow-band ultraviolet B phototherapy in chronic plaque psoriasis. Eur J Dermatol. 2011;21(4):568-572.

10. Kircik L, Bagel J, Korman N, et al. Utilization of narrow-band ultraviolet light B therapy and etanercept for the treatment of psoriasis (UNITE): efficacy, safety, and patient-reported outcomes. J Drugs Dermatol. 2008;7(3):245-253.

11. Wolf P, Hofer A, Legat FJ, et al. Treatment with 311-nm ultraviolet B accelerates and improves the clearance of psoriatic lesions in patients treated with etanercept. Br J Dermatol. 2009;160(1):186-189.

12. Lynde CW, Gupta AK, Guenther L, Poulin Y, Levesque A, Bissonnette R. A randomized study comparing the combination of nbUVB and etanercept to etanercept monotherapy in patients with psoriasis who do not exhibit an excellent response after 12 weeks of etanercept. $J$ Dermatolog Treat. 2012;23(4):261-267.
13. Gambichler T, Tigges C, Scola N, et al. Etanercept plus narrowband ultraviolet $\mathrm{B}$ phototherapy of psoriasis is more effective than etanercept monotherapy at 6 weeks. Br J Dermatol. 2011;164(6):1383-1386.

14. Calzavara-Pinton PG, Sala R, Arisi M, Rossi MT, Venturini M, Ortel B. Synergism between narrowband ultraviolet B phototherapy and etanercept for the treatment of plaque-type psoriasis. $\mathrm{Br} J$ Dermatol. 2013;169(1):130-136.

15. Wolf P, Hofer A, Weger W, Posch-Fabian T, Gruber-Wackernagel A, Legat FJ. $311 \mathrm{~nm}$ ultraviolet B-accelerated response of psoriatic lesions in adalimumab-treated patients. Photodermatol Photoimmunol Photomed. 2011;27(4):186-189.

16. Bagel J. Adalimumab plus narrowband ultraviolet B light phototherapy for the treatment of moderate to severe psoriasis. J Drugs Dermatol. 2011;10(4):366-371.

17. Wolf $P$, Weger W, Legat FJ, et al. Treatment with 311-nm ultraviolet B enhanced response of psoriatic lesions in ustekinumab-treated patients: a randomized intraindividual trial. Br J Dermatol. 2012;166(1):147-153.

18. Park KK, Wu JJ, Koo J. A randomized, 'head-to-head' pilot study comparing the effects of etanercept monotherapy vs. etanercept and narrowband ultraviolet B (NB-UVB) phototherapy in obese psoriasis patients. J Eur Acad Dermatol Venereol. 2013;27(7):899-906.

19. Gordon K, Korman N, Frankel E, et al. Efficacy of etanercept in an integrated multistudy database of patients with psoriasis. $\mathrm{J} \mathrm{Am} \mathrm{Acad}$ Dermatol. 2006;54(3 Suppl 2):S101-S111.

20. Strober B, Gottlieb A, Leonardi C, et al. Levels of response of psoriasis patients with different baseline characteristics treated with etanercept. J Am Acad Dermatol. 2006;54(3 Suppl):AB220.

21. Callis Duffin K, Yeung H, Takeshita J, et al. Patient satisfaction with treatments for moderate-to-severe plaque psoriasis in clinical practice. Br J Dermatol. 2014;170(3):672-680.

22. Zhang M, Brenneman SK, Carter CT, et al. Patient-reported treatment satisfaction and choice of dosing frequency with biologic treatment for moderate to severe plaque psoriasis. Patient Prefer Adherence. 2015;8:777-784

23. Yeung H, Wan J, Van Voorhees AS, et al. Patient-reported reasons for the discontinuation of commonly used treatments for moderate to severe psoriasis. J Am Acad Dermatol. 2013;68(1):64-72.

24. Paul CF, Ho VC, McGeown C, et al. Risk of malignancies in psoriasis patients treated with cyclosporine: a 5 y cohort study. J Invest Dermatol. 2003;120(2):211-216.

25. Patel RV, Clark LN, Lebwohl M, Weinberg JM. Treatments for psoriasis and the risk of malignancy. J Am Acad Dermatol. 2009;60(6): 1001-1017.

26. Di Lernia V, Albertini G. Is antitumour necrosis factor therapy combined with ultraviolet B phototherapy safe? Br J Dermatol. 2010;162(5):1147-1148.

27. Black RJ, Gavin AT. Photocarcinogenic risk of narrowband ultraviolet B (TL-01) phototherapy: early follow-up data. Br J Dermatol. 2006;154(3):566-567.

28. Lee E, Koo J, Berger T. UVB phototherapy and skin cancer risk: a review of the literature. Int J Dermatol. 2005;44(5):355-360.

29. Hearn RM, Kerr AC, Rahim KF, Ferguson J, Dawe RS. Incidence of skin cancers in 3867 patients treated with narrow-band ultraviolet B phototherapy. Br J Dermatol. 2008;159(4):931-935.
Psoriasis: Targets and Therapy

\section{Publish your work in this journal}

Psoriasis: Targets and Therapy is international, peer-reviewed, open access journal focusing on psoriasis, nail psoriasis, psoriatic arthritis and related conditions, identification of therapeutic targets and the optimal use of integrated treatment interventions to achieve improved outcomes

\section{Dovepress}

and quality of life. Visit http://www.dovepress.com/testimonials.php to read real quotes from published authors. 